\title{
Patent analysis of design features of non-cereal crop choppers
}

\author{
Ivan Masienko*, Artem Vasilenko, and Sergey Schevchenko \\ Kuban State Agrarian University named after I.T. Trubilin, Krasnodar, Russia, 350044
}

\begin{abstract}
The paper provides an overview of patent developments mounted and trailed mobile choppers-straw spreaders. Their analysis is carried out and the shortcomings in the process of chopping and field distribution of the non-cereal part of rice crop are indicated. A design of PIRS-2-2 mobile trailed rice straw chopper is proposed, which is to eliminate the disadvantages inherent in the choppers designed for chopping cereal straw. The use of this machine will ensure the required quality of rice straw chopping and uniform distribution of check plots to a given width.
\end{abstract}

In Russia, the Krasnodar Krai is a leading agricultural region in terms of production of winter wheat and rice grain, after the harvesting process in fields and check plots there remains a large amount of non-cereal part of the crop, which must be disposed of or used as soon as possible. The most effective and appropriate way to use the non-crop portion of the harvest of various crops, including rice straw, is to use it as a substance to increase soil fertility by incorporating it into the soil, as scientists have long proved its value as an excellent organic fertilizer [3]. This area of use is especially important at present during the period of insufficient application of valuable manure to the soil due to a decrease in the number of animals in the livestock sector of the region. However, if utilization of cereal straw is not difficult, the utilization of rice straw is complicated due to the specific structure of the stem with hooked scales, which make it difficult to break the bunch, and physical and mechanical features with high silicon content, which leads to increased wear of work surfaces of the coppers' elements, in particular the cutting edges of knives and shear knifes [1].

The technological operations, which differ significantly from similar operations using cereal straw make it difficult to use the non-cereal part of the rice crop as an organic fertilizer and provide high-quality chopping and even incorporation of it into the soil. Currently, both the chopping and the incorporation of the non-cereal part of the rice crop into the soil have not been technically worked out, which does not allow the proposed disposal technology to be used on an industrial scale [2,3]. Therefore, we propose to consider the developments of Russian scientists and inventors of straw choppers - straw spreaders and to analyze their work on chopping and distribution of the non-cereal portion of the rice crop along a check plot.

\footnotetext{
* Corresponding author: ivan.masienko@yandex.ru
} 
There are two ways to chop and distribute the non-cereal part of harvested rice along a check plot: by mounted on a combine harvester chopper simultaneously with rice threshing, and a mobile chopper working separately from the combine with straw picking from a swath [4, 5]. Therefore, we will make a selection and analysis of inventions and utility models of constructions of choppers-spreaders of the non-cereal part of the crop, both mounted on the combine and stand-alone trailed, which are aggregated using a tractor.

An invention design of a straw chopper-spreader of a grain combine harvester, which consists of a body with an installed knife rotor and a spreader equipped with baffle plates is known (Figure 1).
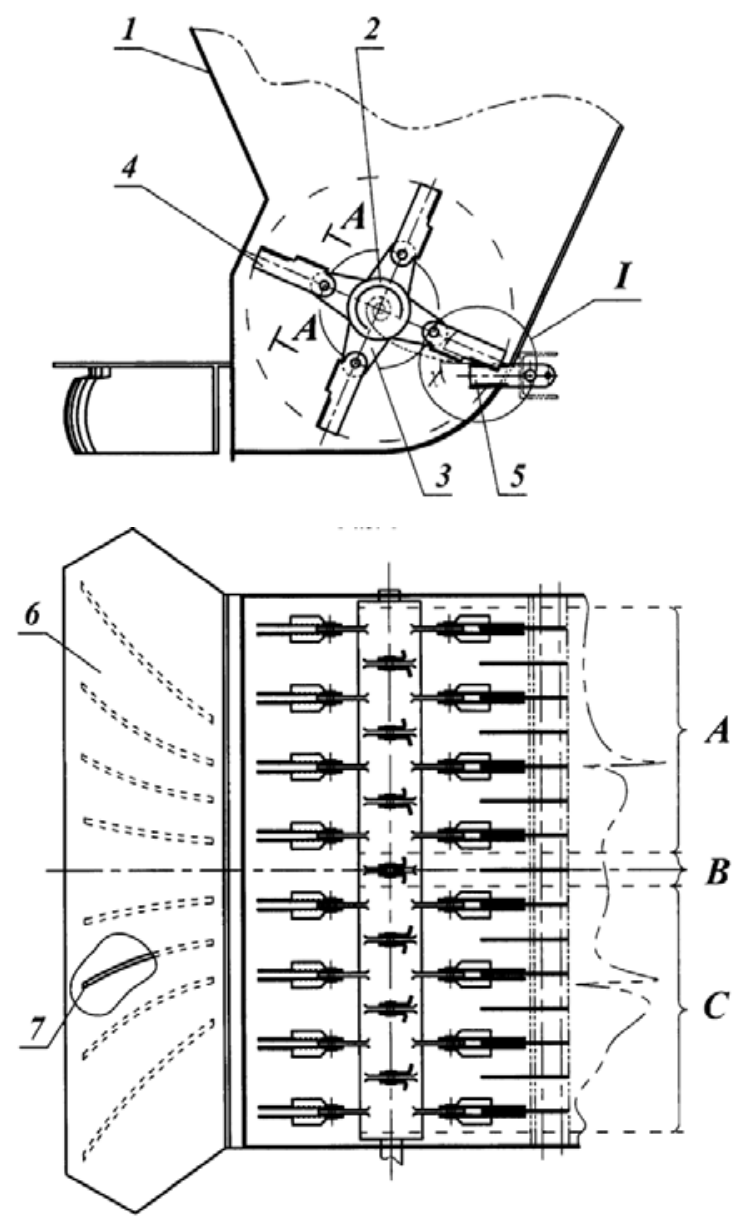

1 - body; 2 - knife rotor; 3 - anchor links; 4 - chopping knife; 5 - counter knife; 6 - straw spreader; 7 baffle plates

Fig. 1. Combine harvester straw shopper-spreader.

In the design of the chopping rotor there are knives with cutting edges, which during rotation interact with fixed counter knives. The chopping knives are made with blades located above the cutting edge at an angle to the plane of the knife. The blades of the cutting knife are oriented away from the center of the rotor. The cutting edges of the counter knives are made concave in the form of a logarithmic spiral with a pole on the axis of rotation of the rotor [6]. 
The present invention for chopping and distribution of straw over the field differs from its predecessors in that the cutting edge of the fixedly mounted counter knives is concave in the form of a logarithmic spiral, and the chopping knives are made with blades located above the cutting edge at an angle to the plane of the knife. At the same time, on each chopping knife located on the side of the rotor periphery, the blade installation angle is less than $90^{\circ}$, and on each chopping knife located on the side of the rotor center, the blade installation angle is more than $90^{\circ}$.

When chopping the non-cereal portion of the rice crop the following disadvantages of the invention were revealed:

- a large number of knives in the design of the chopping drum leads to difficulties in their frequent replacement due to their complete abrasion, which occurs as a result of the high silicon content in the rice straw;

- the difficulties of sharpening the cutting edge of the counter knives with their frequent blunting due to the concave shape made in the form of a logarithmic spiral;

- due to high adhesion of straws and their increased moisture content, distribution of the chopped particles across the entire header width is not uniform.

An invention design of a straw chopper-spreader mounted on a grain combine harvester, which makes it possible to evenly distribute the chopped non-grain part of the crop over the entire width of the header is known. This is achieved by a change in the design of the chopper of the spreading device, which is adapted in such a way that the flow of straw mass leaving the chopper before entering the spreader moves in the direction transverse to the longitudinal axis of the chopper (Figure 2). This is achieved by installing fixed and driven guide elements in the form of centrifugal throwers [7].

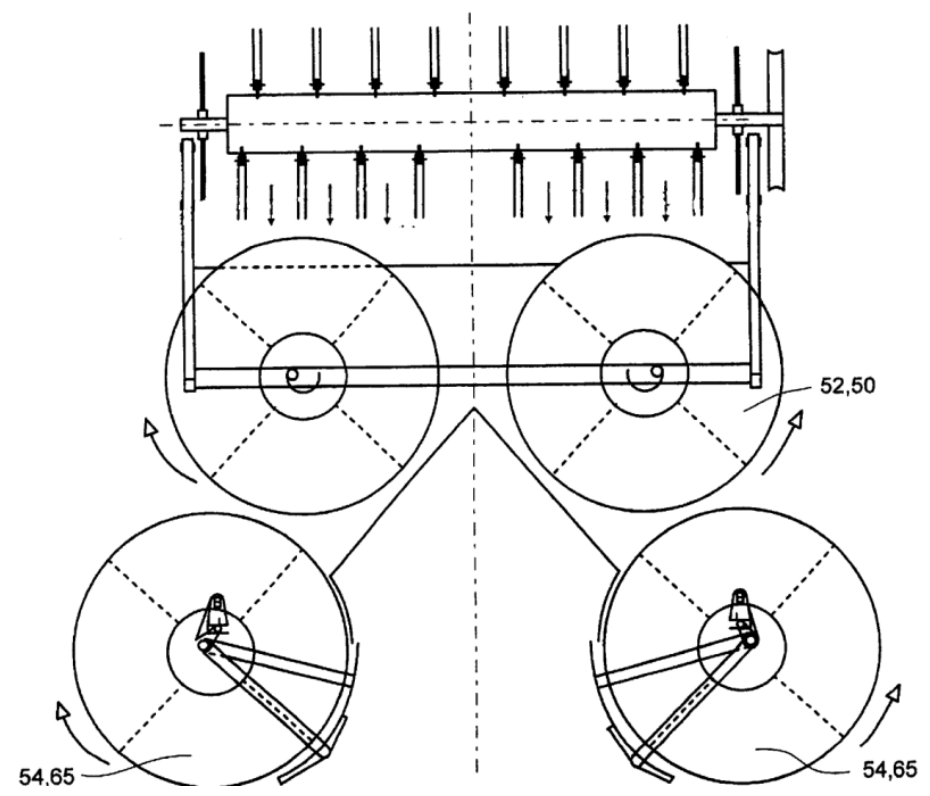

Fig. 2. Cereal grain chopper-spreader.

When grinding the non-cereal portion of the rice crop the following disadvantages of the invention were revealed:

- the complex design of the spreading device leads to frequent node failures, which leads to frequent stops of the combine harvester and to a decrease in its productivity; 
- installation of a spreader in the form of centrifugal throwers increases the mass of the harvester design, which leads to additional slipping and increasing of gauge when harvesting rice in the conditions of swampy check plots;

- installation of a straw spreader leads to a power take-off from a rice harvester, the costs of which are already high when threshing rice and chopping rice straw.

A known construction of a utility model design of a chopper-spreader of non-grain part of the crop from swaths is known (Figure 3). The mobile machine design includes a chassis frame where a feeding device, a collector, compactor and starter rolls, a chopping chamber with a hammer-type chopping drum, an expanding deflector and an unloading mouth are mounted. The design of the trailed chopper - spreader made of rolls differs from analogues in that the unloading mouth of the chopping chamber is equipped with vertical straw-air flow dividers mounted with a step decreasing to the vertical axial plane of the deflector [8].

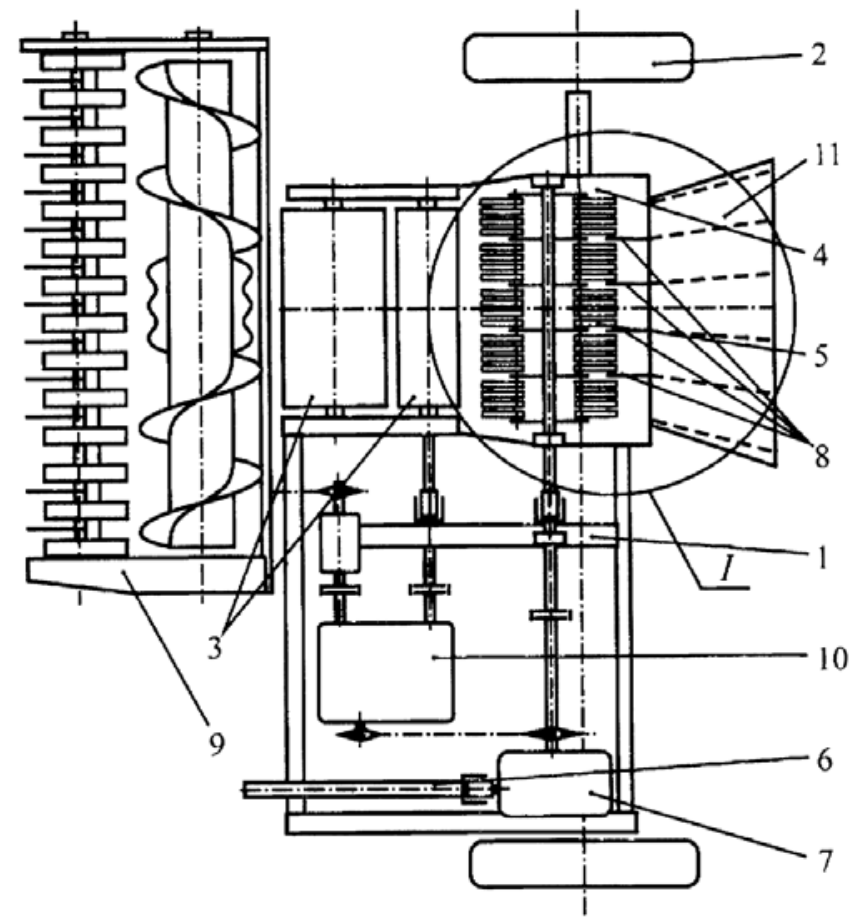

1 - frame; 2 - chassis; 3 - starter and compactor rolls; 4 - chopping chamber; 5 - chopping drum; 6 trailed chopper's drive; 7,10 - gear system; 8 - hammer-type knives; 9 - collector; 11 - deflector

Fig. 3. Cereal crop chopper-spreader.

When chopping the non-cereal part of the rice crop, the following shortcomings of the chopper - spreader from the swaths were revealed:

- a large number of knives in the design of the chopping drum leads to difficulties in their frequent replacement due to their complete abrasion, which occurs as a result of the high silicon content in the rice straw;

- a small width of the deflector leads to frequent clogging and the need to stop the harvesting unit for cleaning;

- due to high adhesion of straws and their increased moisture content, distribution of the chopped particles across the entire header width is not uniform.

The invention design of a collector - chopper of the non-grain part of the crop is known, which is aggregated with a tractor and consists of the main elements: a collector, a frame, a 
feeder, a chopping knife drum, a shear device with fixed knives, a hopper with fertilizer feeds and a deflector (Figure 4). The feeder structure, in turn, consists of starter and compactor rolls installed one above the other, which allow directing the flow of the straw mass and providing persistent cutting. The design of the knife drum consists of chopping knives and blades to create air flow. The fertilizer feeds are installed opposite the window in the upper wall of the distribution deflector. They allow straw to be treated with solid mineral fertilizers to accelerate humification process [9].

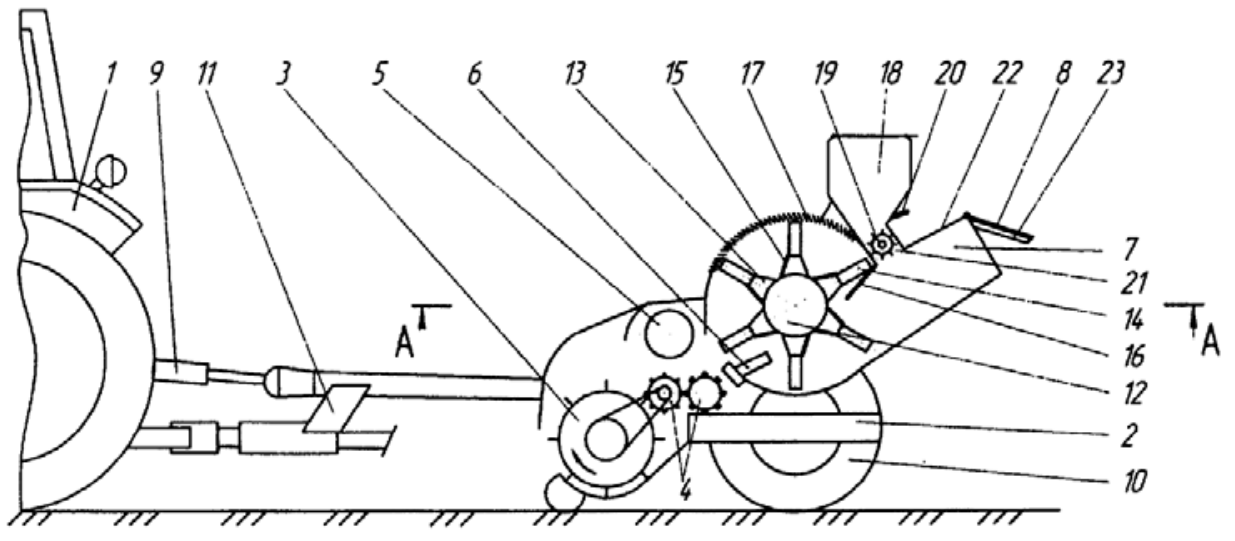

1 - tractor; 2 - frame; 3 - collector; 4 - starter rolls; 5 - compactor roll; 6 - a counter device with fixed knives; 7 - a deflector; 8 - reflective shield; 9 - power take-off; 10 - mover; 11 - connecting beam; 12 - chopping cutting drum; 13 - plate; 14 - knife; 15 - mount; 16 - cutter; 17 - body; 18 - fertilizer feed; 19 - coil; 20 - valve; 21 - window; 22 - spreader body; 23 - baffle plates

Fig. 4. Chopper - spreader of straw.

The present invention for chopping and distributing straw over the field differs from its predecessors because starter and compactor rolls are installed in the receiving chamber, and the fertilizer feeds are opposite the window in the upper wall of the distribution deflector.

When chopping the non-cereal part of the rice crop, the following shortcomings of the chopper - spreader from the swaths were revealed:

- when selecting swaths after threshing rice of large bushiness, the collector gets clogged in front of the compactor rolls;

- solid mineral fertilizers do not have any effect on accelerating the humification of rice straw; for this, rice straw must be treated with liquid mineral fertilizers. Therefore, the use of fertilizer feeds in the design of the grinder - spreader is impractical.

The results of the studies showed that the application of the technology for chopping the non-grain portion of the rice crop with mobile trailed choppers - spreaders is most effective in terms of the quality of the process and is suitable for the harvesting process to separate the operations of threshing rice and utilization of rice straw [6].

When using a mounted chopper - spreader of non-cereal part of the rice crop, the productivity of the harvester is reduced by $30 \%$, fuel consumption is increased by $15 \%$, and because of the high silicon content in the straw, its service life is reduced by a quarter. It should be borne in mind that it is necessary to take about $45-50 \mathrm{~kW}$ of its power from the harvester engine to the chopper - spreader drive. Providing the combine with the function of chopping and spreading the non-grain part of the rice crop leads to a decrease in its productivity and leads to an increase in harvesting time and lower quality of the process $[10,11,12]$.

To eliminate the above-mentioned drawbacks, we propose for the chopping and spreading of the non-grain part of the rice crop on the check plot the design of a trailed rice straw 
chopper (PIRS-2-2), the analogue of which is ZIS-2 grain chopper [10]. A distinctive feature of PIRS-2-2 mobile trailer chopper from the analogue is the addition of a second additional chopping drum-fan to its design, which will ensure separation of the straw flow and, accordingly, unloading of the first chopping drum. Also, this change in design allows the most even distribution of crushed rice straw along the check plot (Figure 5). The grinding drum-fan in its design has 5 cutting knives, which will also reduce the time for servicing them and replacing them during repair [13].

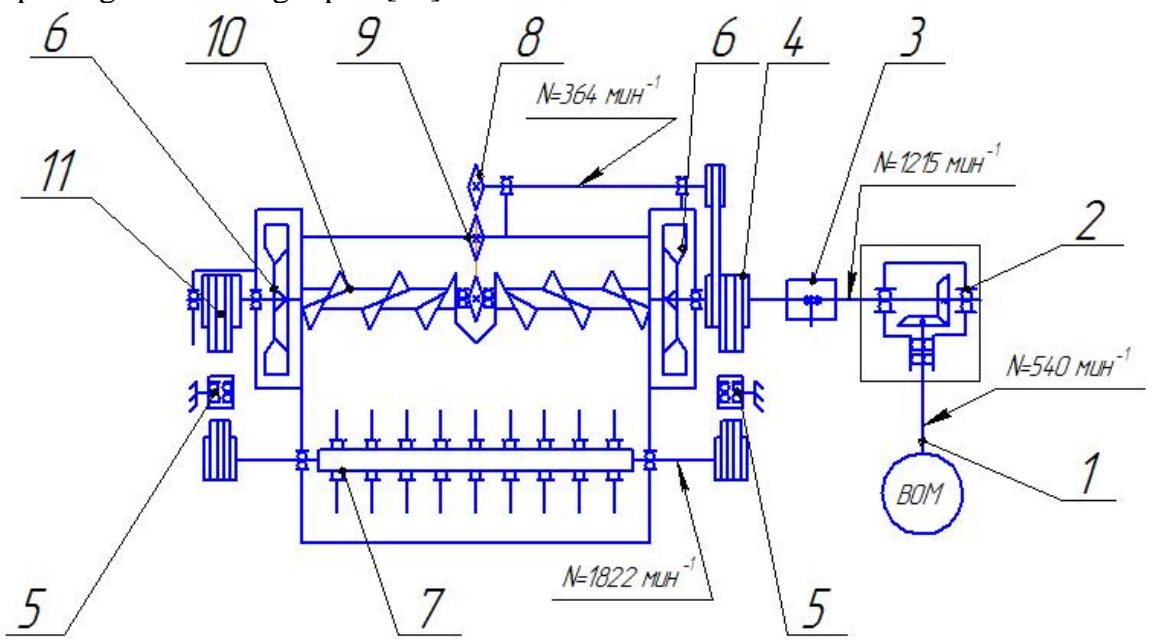

1 - BOM tractor drive; 2 - gear system; 3-chain clutch; 4-belt transmission; 5-tension roller; 6chopper-fan; 7-rotor of the collector apparatus; 8-chain transmission; 9-tightener; 10-sided auger; 11 V-belt transmission for driving an additional chopper-fan.

Fig. 5. Trailed rice straw chopper PIRS-2-2.

The technological process of chopping the non-cereal part of the rice crop with the proposed chopper - spreader PIRS-2-2 takes place in the following sequence. The picking hammers of the rotor 7 raise a swath of rice straw mass, which, passing through the counter knives, is pre-crushed. The pre-crushed straw is sent to the feeding two-sided auger 10, the design of which ensures separation of the straw mass flow into two directions and its supply to the left and right chopper-fans 6 , which finally chop the rice straw to the specified parameters. The crushed mass is directed by the fan blades and the air flow to the nozzles and is evenly distributed over the surface of the check plot to the width of the combine header.

The proposed design of PIRS-2-2 mobile trailer rice chopper will eliminate the disadvantages inherent in the choppers designed for chopping straw of grain crops, reduce the energy consumption of the chopping process, and will improve the quality of the crushed non-grain portion of the rice crop for its subsequent incorporation. The introduction of the proposed design will solve the problem of utilizing the non-grain portion of the rice crop, provide conditions for replenishing the rice systems soil with fresh organic matter, which will ultimately lead to increased soil fertility [14].

\section{References}

1. M. Chebotaryov, I. Masienko, V. Masienko, G. Grigoryan, Recycling rice straw by chopping and splitting with a pin-knife drum. Politematicheskij setevoj jelektronnyj nauchnyj zhurnal Kubanskogo gosudarstvennogo agrarnogo universiteta [Multidisciplinary Network Electronic Scientific Journal of Kuban 
State Agrarian University], 133, pp. 486-497 (2017).

2. M. Chebotaryov, I. Masienko, Technological aspects of utilization of rice straw in rice growing of the Krasnodar Krai, Rice farming, 2 (25), pp. 31-35 (2014).

3. M. Chebotaryov, Digital technologies in rice production, 73, pp. 335-336 (2018).

4. I. Tron, M. Chebotarev, Methods of utilizing the non-cereal portion of rice in the conditions of the Krasnodar Krai, 73, pp. 415-419 (2018).

5. G. Zelenskiy, M. Chebotaryov, T. Logoyda, Improving the technology of rice cultivation in sanitary zones. Trudy Kubanskogo gosudarstvennogo agrarnogo universiteta [Proceedings of the Kuban State Agrarian University], 74, pp. 53-57 (2018).

6. M. Yagolskiy, S. Rodimtsev, Chopper - straw spreader of a combine harvester, Patent 2647902 of the Russian Federation 9, (2018).

7. S. Terede, Chopper - spreader, Patent 2442314 of the Russian Federation 5, (2012).

8. V. Sysuev, Chopper - straw spreader from rolls, Patent 20815 of the Russian Federation 34, (2001).

9. V. Sysuev, Sorter - straw chopper. Patent 2335884 of the Russian Federation 29, (2008).

10. M. Chebotaryov, E. Shapiro, A. Taran, Determining the total number of rice harvesters with a given guarantee probability, Rural mechanic, 7-8, pp. 71-86 (2018).

11. M. Chebotaryov, E. Shapiro, A. Taran, Calculation of the guaranteed number of rice harvesters in the main technological link of the harvesting and transport complex, Rice Production, 4 (41), pp. 56-59 (2018).

12. M. Chebotaryov, Current status and development prospects of the system of machines for rice growing, pp. 239-245 (2016).

13. M. Chebotaryov, I. Masienko, G. Grigoryan, V. Gritsunov, Trailed chopper of rice straw PIRS-2-2, Rural machine operator, 10, pp. 38-39 (2018).

14. I. Masienko, Development prospects of mobile rice straw crushers. In the collection: E3S Web of Conferences, c. 00022, (2019). 